\title{
Discrete bipolar universal integrals
}

\author{
Salvatore Greco $^{1}$, Radko Mesiar ${ }^{2}$, and Fabio Rindone ${ }^{1}$ \\ 1 Department of Economics and Business, \\ University of Catania, Corso Italia 55, 95129 Catania, Italy \\ salgreco@unict.it,frindone@unict.it \\ 2 Department of Mathematics and Descriptive Geometry, \\ Faculty of Civil Engineering, Slovak University of Technology, Bratislava, Slovakia \\ and \\ Institute of Theory of Information and Automation, \\ Czech Academy of Sciences, Prague, Czech Republic \\ radko.mesiar@stuba.sk
}

\begin{abstract}
The concept of universal integral, recently proposed, generalizes the Choquet, Shilkret and Sugeno integrals. Those integrals admit a discrete bipolar formulation, useful in those situations where the underlying scale is bipolar. In this paper we propose the concept of discrete bipolar universal integral, in order to provide a common framework for bipolar discrete integrals, including as special cases the discrete Choquet, Shilkret and Sugeno bipolar integrals. Moreover we provide two different axiomatic characterizations of the proposed discrete bipolar universal integral.
\end{abstract}

Key words: Choquet integral; Sugeno integral; Shilkret integral; universal integral; bipolar integral.

\section{Introduction}

Recently, a concept of universal integral has been proposed [26]. The universal integral generalizes the Choquet integral [4], the Sugeno integral [35] and the Shilkret integral [33]. Moreover, in [23, 24] a formulation of the universal integral with respect to a level dependent capacity has been proposed, in order to generalize the level-dependent Choquet integral [17], the level-dependent Shilkret integral [3] and the level-dependent Sugeno integral [29]. The Choquet, Shilkret and Sugeno integrals admit a discrete bipolar formulation, useful in those situations where the underlying scale is bipolar $[11,12,18,16,20]$. In this paper we introduce and characterize the discrete bipolar universal integral, which generalizes the discrete Choquet, Shilkret and Sugeno bipolar integrals.

Let us briefly describe the economic motivations of this paper. In the last three/four decades non-additive integrals - i.e. those integrals based on monotone measures, non necessarily additive - have been applied to many fields of Decision Analysis. 
For example, in the field of multiple-criteria decision aid (MCDA), the use of non-additive integrals (called fuzzy integrals) is nowadays pervasive [7, 13]. The motivation is due, essentially, to the fact that non-additive integrals, when used as aggregation functions, allow for a natural representation of the interaction of criteria.

In decision making under risk and uncertainty for a large time, the dominant model has been the Expected Utility Theory (EUT) [38]. The EUT value function is based on the Lebesgue integral, but the additivity of this integral when applied to real choice (especially in economics) leads to unrealistic conclusions (see e.g. [1, 5, 22, 36]). For these motivations the development of new theories, called non-EUT theories, and based on non-additive integrals has increased very fast (for a seminal survey we recommend [34]). In decision making under risk and uncertainty, the Choquet integral has firstly received an axiomatic characterization [31] and then has been successfully applied to economic models of decision: over all we remember the Choquet Expected Utility (CEU) of Schmeidler and Gilboa [6, 32] and the Cumulative Prospect Theory of Kahneman and Tversky [37].

Very recently, one of the most interesting lines of research has concerned the bipolarity of choices: the decision maker individuates a reference point and, then separates gains (alternatives greater than the reference point) from losses (alternatives smaller than the reference point); symmetric choices with respect to the reference point are considered. Regarding a general discussion on the use of bipolarity the reader is referred to [10, 28], while regarding the generalization of well known integrals, used in MCDM, to the bipolar case, the reader is referred to $[14,20]$. Also in decision under risk and uncertainty, the necessity of new tools able to model the bipolarity has emerged [27, 39]. In [21] the bipolar Choquet integral of Grabisch and Labreuche [12] has been used in order to obtain a bipolar generalization of CPT.

The paper is organized as follows. In section 2 we introduce the basic concepts. In section 3 we define and characterize the bipolar universal integral. In section 4 we give an illustrative example of a bipolar universal integral which is neither the Choquet nor Sugeno or Shilkret type. Section 5 shows how the discrete universal integral can be also characterized in terms of a family of aggregation functions satisfying a set of desired axioms. Finally, in section 6 , we present conclusions.

\section{Basic concepts}

For the sake of simplicity, in this work we present the results in a multiple criteria decision making setting. Given a set of criteria $X=\{1, \ldots, n\}$, an alternative $\boldsymbol{x}$ can be identified with a score vector $\boldsymbol{x}=\left(x_{1}, \ldots, x_{n}\right) \in[-\infty,+\infty]^{n}$, being $x_{i}$ the evaluation of $\boldsymbol{x}$ with respect to the $i$ th criterion. Without loss of generality, in the following we consider the bipolar scale $[-1,1]$ to expose our results, so that $\boldsymbol{x} \in[-1,1]^{n}$. For all $\boldsymbol{x}=\left(x_{1} \ldots, x_{n}\right) \in[-1,1]^{n}$, the set $\left\{i \in X \mid x_{i} \geq t\right\}, t \in[0,1]$, is briefly indicated with $\{\boldsymbol{x} \geq t\}$. Similar meaning 
have the symbols $\{\boldsymbol{x} \leq t\},\{\boldsymbol{x}>t\}$ and $\{\boldsymbol{x}<t\}$. For all $\boldsymbol{x}, \boldsymbol{y} \in[-1,1]^{n}$ we say that $\boldsymbol{x}$ dominates $\boldsymbol{y}$ and we write $\boldsymbol{x} \succeq \boldsymbol{y}$, if $x_{i} \geq y_{i}, i=1, \ldots, n$. Let us consider the set $\mathcal{Q}=\left\{(A, B) \in 2^{X} \times 2^{X} \mid A \cap B=\emptyset\right\}$ of all disjoint pairs of subsets of $X$, see [11]. With respect to the binary relation $\precsim$ on $\mathcal{Q}$ defined as $(A, B) \precsim(C, D)$ iff $A \subseteq C$ and $B \supseteq D, \mathcal{Q}$ is a lattice, i.e., a partially ordered set in which any two elements have a unique supremum $(A, B) \vee(C, D)=(A \cup C, B \cap D)$ and a unique infimum $(A, B) \wedge(C, D)=(A \cap C, B \cup D)$. For all $(A, B) \in \mathcal{Q}$ the vector $\mathbf{1}_{(A, B)} \in[-1,1]^{n}$ is the vector whose $i$ th component equals 1 if $i \in A$, equals -1 if $i \in B$ and equals 0 else. A bipolar aggregation function $f:[-1,1]^{n} \rightarrow[-1,1]$ is a function such that $f(\boldsymbol{x}) \geq f(\boldsymbol{y})$ whenever $\boldsymbol{x} \succeq \boldsymbol{y}$ and $f\left(\mathbf{1}_{(X, \emptyset)}\right)=1, f\left(\mathbf{1}_{(\emptyset, X)}\right)=-1$ and $f\left(\mathbf{1}_{(\emptyset, \emptyset)}\right)=0$. We indicate with $\mathcal{A}_{[-1,1]^{n}}$ the set of aggregation functions on $[-1,1]^{n}$.

Definition 1 A function $\mu_{b}: \mathcal{Q} \rightarrow[-1,1]$ is a (normalized) bi-capacity [11, 12, 18] on $X$ if

$$
\begin{aligned}
& -\mu_{b}(\emptyset, \emptyset)=0, \mu_{b}(X, \emptyset)=1 \text { and } \mu_{b}(\emptyset, X)=-1 ; \\
& -\mu_{b}(A, B) \leq \mu_{b}(C, D) \text { for all }(A, B),(C, D) \in \mathcal{Q} \text { such that }(A, B) \precsim(C, D) .
\end{aligned}
$$

Let us note that, by the sake of simplicity, we use the abbreviated form $\mu(A, B)$ instead of $\mu((A, B))$.

Definition 2 The bipolar Choquet integral of $\boldsymbol{x}=\left(x_{1}, \ldots, x_{n}\right) \in[-1,1]^{n}$ with respect to the bi-capacity $\mu_{b}$ is given by [11, 12, 18]:

$$
C h_{b}\left(\boldsymbol{x}, \mu_{b}\right)=\int_{0}^{1} \mu_{b}(\{\boldsymbol{x}>t\},\{\boldsymbol{x}<-t\}) d t .
$$

The bipolar Choquet integral of $\boldsymbol{x}=\left(x_{1}, \ldots, x_{n}\right) \in[-1,1]^{n}$ with respect to the bi-capacity $\mu_{b}$ can be rewritten as

$$
C h_{b}\left(\boldsymbol{x}, \mu_{b}\right)=\sum_{i=1}^{n}\left(\left|x_{\sigma(i)}\right|-\left|x_{\sigma(i-1)}\right|\right) \mu_{b}\left(\left\{\boldsymbol{x} \geq\left|x_{\sigma(i)}\right|\right\},\left\{\boldsymbol{x} \leq-\left|x_{\sigma(i)}\right|\right\}\right),
$$

being $\sigma: X \rightarrow X$ any permutation of indexes such that $0=\left|x_{\sigma(0)}\right| \leq\left|x_{\sigma(1)}\right| \leq \ldots \leq$ $\left|x_{\sigma(n)}\right|$. Let us note that to ensure that $(\{\boldsymbol{x} \geq|t|\},\{\boldsymbol{x} \leq-|t|\}) \in \mathcal{Q}$ for all $t \in \mathbb{R}$, we adopt the convention - which will be maintained trough all the paper - that in the case of $t=0$ the inequality $x_{j} \leq 0$ is to be understood as $x_{j}<0$.

In this paper we use the symbol $\vee$ to indicate the maximum and $\wedge$ to indicate the minimum. The symmetric maximum of two elements - introduced and discussed in $[8,9]$ - is defined by the following binary operation:

$$
a \otimes b= \begin{cases}-(|a| \vee|b|) & \text { if } b \neq-a \text { and either }|a| \vee|b|=-a \text { or }=-b \\ 0 & \text { if } b=-a \\ |a| \vee|b| & \text { else. }\end{cases}
$$


Alternatively the symmetric maximum of $a, b \in \mathbb{R}$ can be written as

$$
a \otimes b=\operatorname{sign}(a+b)(|a| \vee|b|) .
$$

The symmetric minimum of two elements $[8,9]$ is defined as:

$$
a \otimes b= \begin{cases}-(|a| \wedge|b|) & \text { if } \operatorname{sign}(b) \neq \operatorname{sign}(a) \\ |a| \wedge|b| & \text { else. }\end{cases}
$$

Alternatively the symmetric minimum of $a, b \in \mathbb{R}$ can be written as

$$
a \oplus b=\operatorname{sign}(a \cdot b)(|a| \wedge|b|) .
$$

In [30] it has been shown that on the domain $[-1,1]$ the symmetric maximum coincides with two recent symmetric extensions of the Choquet integral, the balancing Choquet integral and the fusion Choquet integral, when they are computed with respect to the strongest capacity (i.e. the capacity which takes the value zero on the empty set and one elsewhere). However, the symmetric maximum of a set $X$ cannot be defined without any ambiguity, being $\oslash$ non associative. Suppose that $X=\{3,-3,2\}$, then $(3 \otimes-3) \oslash 2=2$ or $3 \otimes(-3 \otimes 2)=0$, depending on the order of aggregation. Several possible extensions of the symmetric maximum for dimension $n, n>2$, have been proposed (see $[9,15]$ and also the relative discussion in [30]). One of these extensions is based on the splitting rule applied to the maximum and to the minimum as described in the following. Given $X=\left\{x_{1}, \ldots, x_{m}\right\} \subseteq \mathbb{R}$, the bipolar maximum of $X$, shortly $\bigvee^{b} X$, is defined as

$$
\bigvee^{b} X=(\bigvee X) \otimes(\bigwedge X)
$$

In the same way and for an infinite set $X$, it is possible to define the concept of bipolar supremum of $X$, $\sup ^{b i p} X$, as the symmetric maximum applied to the supremum and the infimum of $X$ :

$$
\sup ^{b i p} X=\sup X \otimes \inf X,
$$

with the convention that $\pm \infty \otimes l= \pm \infty$ for all $l \in \mathbb{R}$ and $+\infty \otimes(-\infty)=0$.

Definition 3 The bipolar Shilkret integral of $\boldsymbol{x}=\left(x_{1}, \ldots, x_{n}\right) \in[-1,1]^{n}$ with respect to the bi-capacity $\mu_{b}$ is given by [20]:

$$
S h_{b}\left(\boldsymbol{x}, \mu_{b}\right)=\bigvee_{i \in X}^{b}\left\{\left|x_{i}\right| \cdot \mu_{b}\left(\left\{\boldsymbol{x} \geq\left|x_{i}\right|\right\},\left\{\boldsymbol{x} \leq-\left|x_{i}\right|\right\}\right)\right\} .
$$

Definition 4 The bipolar Sugeno integral of $\boldsymbol{x}=\left(x_{1}, \ldots, x_{n}\right) \in[-1,1]^{n}$ with respect to the bi-capacity $\mu_{b}$ on $X$ is given by [20]:

$$
S u_{b}\left(\boldsymbol{x}, \mu_{b}\right)=\bigvee_{i \in X}^{b}\left\{\left|x_{i}\right| \otimes \mu_{b}\left(\left\{\boldsymbol{x} \geq\left|x_{i}\right|\right\},\left\{\boldsymbol{x} \leq-\left|x_{i}\right|\right\}\right)\right\}
$$

The discrete bipolar Choquet Shilkret and Sugeno integrals defined in (1), (4) and (5) are aggregation functions from $\mathcal{A}_{[-1,1]^{n}}$ and they have been axiomatized in [20]. Next sections provide a general framework for these discrete bipolar fuzzy integrals. 


\section{Discrete universal integrals and discrete bipolar universal integrals}

In order to define universal integrals it is necessary to introduce the concept of semicopula [2].

Definition 5 A semicopula is a function $\otimes:[0,1]^{2} \rightarrow[0,1]$ such that for all $x, y, t$ and $z \in[0,1]$ the following axioms are satisfied:

- monotonicity: $x \otimes y \leq t \otimes z$, whenever $x \leq t$ and $y \leq z$;

- 1 is a neutral element: $1 \otimes x=x \otimes 1=x$.

Note that a semicopula has 0 as annihilator. Indeed $0 \leq a \otimes 0 \leq 1 \otimes 0=0$ and $0 \leq 0 \otimes a \leq 0 \otimes 1=0$. A commutative and associative semicopula is called a $t$-norm. Typical examples of t-norms are the minimum $(\wedge)$, the product $(\cdot)$, and the Lukasiewicz t-norm defined by $T_{L}(x, y)=(x+y-1) \vee 0$.

The concept of universal integrals, which can be defined for arbitrary monotone measures on arbitrary measurable spaces $(S ; \mathcal{A})$ and for arbitrary measurable functions $f: S \rightarrow[0, \infty]$, was axiomatically introduced in [26]. Here we use the concept of $[0,1]$-valued discrete universal integral [25], defined on the union of all measurable spaces $\left(X_{n}, 2^{X_{n}}\right)$, where the finite space $X_{n}=\{1, \ldots, n\}$ is considered for all $n \in \mathbb{N}$ and is equipped with the $\sigma$-algebra $2^{X_{n}}$. Functions from $X_{n}$ to $[0,1]$ are identified with $n$-dimensional vectors of $[0,1]^{n}$. Let $M_{n}$ denotes the set of all capacities on $X_{n}$ i.e., the set of all monotone set functions $m: 2^{X_{n}} \rightarrow[0,1]$ such that $m(\emptyset)=0$ and $m\left(X_{n}\right)=1$. For all $n \in \mathbb{N}$, and $E \subseteq X_{n}$ the characteristic function $1_{E}$ is identified with the vector $\mathbf{1}_{E} \in[0,1]^{n}$ whose $i$ th component equals 1 if $i \in E$ and equals 0 otherwise.

Definition 6 A function $I: \bigcup_{n \in \mathbb{N}}\left(M_{n} \times[0,1]^{n}\right) \rightarrow[0,1]$ is a $[0,1]$-valued discrete universal integral [25] if it satisfies the following axioms:

(I1) $I(m, \boldsymbol{x})$ is nondecreasing with respect to $m$ and with respect to $\boldsymbol{x}$;

(I2) $I\left(m, \mathbf{1}_{E}\right)=m(E)$, for all $n \in \mathbb{N}, m \in M_{n}$ and $E \subseteq X_{n}$;

(I3) $I\left(m, c \cdot \mathbf{1}_{X_{n}}\right)=c$, for all $n \in \mathbb{N}, m \in M_{n}$ and $c \in[0,1]$

(I4) $I\left(m_{1}, \boldsymbol{x}_{1}\right)=I\left(m_{2}, \boldsymbol{x}_{2}\right)$, for all $\left(m_{1}, \boldsymbol{x}_{1}\right) \in M_{n_{1}} \times[0,1]^{n_{1}},\left(m_{2}, \boldsymbol{x}_{2}\right) \in M_{n_{2}} \times[0,1]^{n_{2}}$, $n_{1}, n_{2} \in \mathbb{N}$, such that for all $t \in[0,1], m_{1}\left(\left\{\boldsymbol{x}_{1} \geq t\right\}\right)=m_{2}\left(\left\{\boldsymbol{x}_{2} \geq t\right\}\right)$.

In [25] the following proposition has been stated.

Proposition 1 Let I be a [0,1]-valued discrete universal integral. Then there exists a semicopula $\otimes$ such that we have $I\left(m, c \cdot \mathbf{1}_{A}\right)=c \otimes m(A)$ for all $n \in \mathbb{N}, m \in M_{n}$, $c \in[0,1]$ and $A \subseteq X_{n}$.

Due to Proposition 1 in Definition 6, axioms (I2) and (I3) can be equivalently substituted with the following axiom

(I5) there exists a semicopula $\otimes$ such that $I\left(m, c \cdot \mathbf{1}_{A}\right)=c \otimes m(A)$ for all $n \in \mathbb{N}$, $m \in M_{n}, c \in[0,1]$ and $A \subseteq X_{n}$.

The following theorem represents an alternative axiomatic characterization of the $[0,1]$-valued discrete universal integral. 
Theorem 1 A function $I: \bigcup_{n \in \mathbb{N}}\left(M_{n} \times[0,1]^{n}\right) \rightarrow[0,1]$ is a $[0,1]$-valued discrete universal integral if and only if it satisfies the following axioms:

(I5) there exists a semicopula $\otimes$ such that $I\left(m, c \cdot \mathbf{1}_{A}\right)=c \otimes m(A)$ for all $n \in \mathbb{N}$, $m \in M_{n}, c \in[0,1]$ and $A \subseteq X_{n}$.

(I6) $I\left(m_{1}, \boldsymbol{x}_{1}\right) \geq I\left(m_{2}, \boldsymbol{x}_{2}\right)$ for all $\left(m_{1}, \boldsymbol{x}_{1}\right) \in M_{n_{1}} \times[0,1]^{n_{1}}$ and $\left(m_{2}, \boldsymbol{x}_{2}\right) \in M_{n_{2}} \times$ $[0,1]^{n_{2}}, n_{1}, n_{2} \in \mathbb{N}$, such that for all $t \in[0,1], m_{1}\left(\left\{\boldsymbol{x}_{1} \geq t\right\}\right) \geq m_{2}\left(\left\{\boldsymbol{x}_{2} \geq t\right\}\right)$.

Proof. The sufficiency part is obvious since it is easily checked that (I6) implies (I1) and (I4) while (I5) implies (I2) and (I3). Now we prove the necessity part.

Let us suppose that axioms (I1)-(I4) hold. Axiom (I5) is true by Proposition 1 and, then, we only have to prove axiom (I6). Let us suppose that for some $n_{1}, n_{2} \in \mathbb{N}$, $X_{n_{1}}=\left\{c_{1}, \ldots, c_{n_{1}}\right\}$ and $X_{n_{2}}=\left\{d_{1}, \ldots, d_{n_{2}}\right\}$ are two sets of disjoint criteria, i.e. $X_{n_{1}} \cap X_{n_{2}}=\emptyset$. Consider $\left(m_{1}, \boldsymbol{x}\right) \in M_{n_{1}} \times[0,1]^{n_{1}}$ and $\left(m_{2}, \boldsymbol{y}\right) \in M_{n_{2}} \times[0,1]^{n_{2}}$ such that for all $t \in[0,1], m_{1}(\{\boldsymbol{x} \geq t\}) \geq m_{2}(\{\boldsymbol{y} \geq t\})$. If $\boldsymbol{x}=\left(x_{1}, \ldots, x_{n_{1}}\right)$ and $\boldsymbol{y}=\left(y_{1}, \ldots, y_{n_{2}}\right)$ let us consider vector $\boldsymbol{z}=\left(x_{1}, \ldots, x_{n_{1}}, y_{1}, \ldots, y_{n_{2}}\right) \in[0,1]^{n_{1}+n_{2}}$ and the two capacities $\bar{m}, \underline{m}: 2^{X_{n_{1}} \cup X_{n_{2}}} \rightarrow[0,1]$ defined for all $E \subseteq X_{n_{1}} \cup X_{n_{2}}$ by

$$
\bar{m}(E)=\vee\left\{m_{1}\left(E \cap X_{1}\right), m_{2}\left(E \cap X_{2}\right)\right\}
$$

and

$$
\underline{m}(E)=\wedge\left\{m_{1}\left(E \cap X_{1}\right), m_{2}\left(E \cap X_{2}\right)\right\} .
$$

By axiom (I4) we have that $I\left(m_{1}, \boldsymbol{x}_{1}\right)=I(\bar{m}, \boldsymbol{z})$ and $I\left(m_{2}, \boldsymbol{x}_{2}\right)=I(\underline{m}, \boldsymbol{z})$ and by axiom (I1), being $\bar{m} \geq \underline{m}, I(\bar{m}, \boldsymbol{z}) \geq I(\underline{m}, \boldsymbol{z})$. Note that the condition $X_{n_{1}} \cap X_{n_{2}}=\emptyset$ does not represent a limitation, since by axiom (I4), the value $I(m, \boldsymbol{x})$ does not depend on the underlying measurable space $\left(X_{n}, 2^{X_{n}}\right)$ but only on the values of $m \in M_{n}$ on the correspondent subsets $\{\boldsymbol{x} \geq t\}$ from $X_{n}$.

Remark 1 Observe that the necessity in Theorem 1 can be shown also alternatively by means of [26, Proposition 2.7].

Now let us generalize the concept of discrete universal integral from the scale $[0,1]$ to the symmetric scale $[-1,1]$ by extending definition 6 and generalizing the definition of bipolar universal integrals given in [19]. Again the finite space $X_{n}$ is considered for all $n \in \mathbb{N}$ and similarly, the lattice $\mathcal{Q}$, which will be denoted with $\mathcal{Q}_{n}$ and the set of all normalized bi-capacities on $X_{n}$, which will be denoted with $M_{n}^{b}$. Functions from $X_{n}$ to $[-1,1]$ are identified with $n$-dimensional vectors of $[-1,1]^{n}$.

Definition 7 A function $I_{b}: \bigcup_{n \in \mathbb{N}}\left(M_{n}^{b} \times[-1,1]^{n}\right) \rightarrow[-1,1]$ is a $[-1,1]-$ valued discrete bipolar universal integral if it satisfies the following axioms:

(U1) $I_{b}\left(\mu_{b}, \boldsymbol{x}\right)$ is nondecreasing with respect to $\mu_{b}$ and with respect to $\boldsymbol{x}$;

(U2) $I_{b}\left(\mu_{b}, \mathbf{1}_{(A, B)}\right)=\mu_{b}(A, B)$, for all $n \in \mathbb{N}, \mu_{b} \in M_{n}^{b}$ and $(A, B) \in \mathcal{Q}_{n}$;

(U3) $I_{b}\left(\mu_{b}, c \cdot \mathbf{1}_{\left(X_{n}, \emptyset\right)}\right)=-I_{b}\left(\mu_{b}, c \cdot \mathbf{1}_{\left(\emptyset, X_{n}\right)}\right)=c$, for all $n \in \mathbb{N}, \mu_{b} \in M_{n}^{b}, c \in[0,1]$;

(U4) if $I_{b}\left(\mu_{b_{1}}, \mathbf{1}_{\left(A_{1}, B_{1}\right)}\right)=-I_{b}\left(\mu_{b_{2}}, \mathbf{1}_{\left(A_{2}, B_{2}\right)}\right)$ for some $n_{1}, n_{2} \in \mathbb{N}, \mu_{b_{1}} \in M_{n_{1}}^{b}, \mu_{b_{2}} \in$ $M_{n_{2}}^{b},\left(A_{1}, B_{1}\right) \in \mathcal{Q}_{n_{1}}$ and $\left(A_{2}, B_{2}\right) \in \mathcal{Q}_{n_{2}}$, then

$$
I_{b}\left(\mu_{b_{1}}, c \cdot \mathbf{1}_{\left(A_{1}, B_{1}\right)}\right)=-I_{b}\left(\mu_{b_{2}}, c \cdot \mathbf{1}_{\left(A_{2}, B_{2}\right)}\right) ;
$$


(U5) $I_{b}\left(\mu_{b_{1}}, \boldsymbol{x}_{1}\right)=I_{b}\left(\mu_{b_{2}}, \boldsymbol{x}_{2}\right)$ for all pairs $\left(\mu_{b_{1}}, \boldsymbol{x}_{1}\right) \in M_{n_{1}}^{b} \times[-1,1]^{n_{1}}$ and $\left(\mu_{b_{2}}, \boldsymbol{x}_{2}\right) \in$ $M_{n_{2}}^{b} \times[-1,1]^{n_{2}}, n_{1}, n_{2} \in \mathbb{N}$, such that for all $\left.\left.t \in\right] 0,1\right]$,

$$
\mu_{b_{1}}\left(\left\{\boldsymbol{x}_{1} \geq t\right\},\left\{\boldsymbol{x}_{1} \leq-t\right\}\right)=\mu_{b_{2}}\left(\left\{\boldsymbol{x}_{2} \geq t\right\},\left\{\boldsymbol{x}_{2} \leq-t\right\}\right) .
$$

The following proposition holds

Proposition 2 Let $I_{b}: \bigcup_{n \in \mathbb{N}}\left(M_{n}^{b} \times[-1,1]^{n}\right) \rightarrow[-1,1]$ be a $[-1,1]$-valued discrete bipolar universal integral. Then there exists a semicopula $\otimes$ such that for all $n \in \mathbb{N}$, $\mu_{b} \in M_{n}^{b}, c \in[0,1]$ and $(A, B) \in Q_{n}$,

$$
I_{b}\left(\mu_{b}, c \cdot \mathbf{1}_{(A, B)}\right)=\operatorname{sign}\left(\mu_{b}(A, B)\right)\left(c \otimes\left|\mu_{b}(A, B)\right|\right) .
$$

Proof. First of all let us observe that for any $c \in[0,1]$ and for any $n \in \mathbb{N}, n \geq 2$ we can define a bi-capacity $\mu_{b} \in \mathcal{Q}_{n}$ by setting $\mu_{b}(A, B)=0$ if $A \cap B \neq \emptyset, \mu_{b}(A, \emptyset)=c$ and $\mu_{b}(\emptyset, A)=-c$ if $A \in 2^{X_{n}} \backslash\left\{\emptyset, X_{n}\right\}$.

Now, for all $a, c \in[0,1]$, let us define the function $a \otimes c=I_{b}\left(\mu, a \mathbf{1}_{(A, B)}\right)=$ $-I_{b}\left(\mu^{*}, a \mathbf{1}_{\left(A^{*}, B^{*}\right)}\right)$, for all $n, n^{*} \in \mathbb{N}, \mu_{b} \in M_{n}^{b}, \mu_{b}^{*} \in M_{n^{*}}^{b}$ and $(A, B) \in \mathcal{Q}_{n},\left(A^{*}, B^{*}\right) \in$ $\mathcal{Q}_{n^{*}}$ such that $\mu_{b}(A, B)=c$ and $\mu_{b}^{*}\left(A^{*}, B^{*}\right)=-c$. Due to axiom (U4) and (U5) and to the remark at the beginning of the proof,this definition is well posed.

Now we check that $\otimes$ is a semicopula, starting by proving monotonicity. Suppose that $a_{1}, a_{2} \in[0,1]$ with $a_{1}<a_{2}$, then by monotonicity of $I_{b}$ we have that $a_{1} \otimes c=$ $I_{b}\left(\mu_{b}, a_{1} \mathbf{1}_{(A, B)}\right) \leq a_{2} \otimes c=I_{b}\left(\mu_{b}, a_{2} \mathbf{1}_{(A, B)}\right)$ for all $c \in[0,1]$ and for some $n \in \mathbb{N}$, $\mu_{b} \in M_{n}^{b}$ such that $\mu_{b}(A, B)=c$. If $b_{1}, b_{2} \in[0,1]$ with $b_{1}<b_{2}$ there exists an $n \in \mathbb{N}$, $n \geq 3, \mu_{b} \in M_{n}^{b}, E \subseteq F \subseteq X_{n}$ such that $\mu_{b}(E, \emptyset)=b_{1}$ and $\mu_{m}(F, \emptyset)=b_{2}$ (see the beginning of the proof). Now, for all $a \in[0,1]$ we have that $a \otimes b_{1}=I_{b}\left(\mu_{b}, a \mathbf{1}_{(E, \emptyset)}\right)=\leq$ $a \otimes b_{2}=I_{b}\left(\mu_{b}, a \mathbf{1}_{(F, \emptyset)}\right)$, by monotonicity of $I_{b}$. We conclude that $\otimes$ is nondeacreasing in both components.

Now we prove that 1 is a neutral element for $\otimes$. For all $a \in[0,1], a \otimes 1=$ $I_{b}\left(\mu_{b}, a \mathbf{1}_{X_{n}}\right)=a$ by $(\mathrm{U} 3)$ and $1 \otimes a=I_{b}\left(\mu_{b}, \mathbf{1}_{(A, B)}\right)=a$ for all $n \in \mathbb{N}$, and $\mu_{b} \in M_{n}^{b}$ such that $\mu_{b}(A, B)=a$, by (U2).

Finally, it is obvious, by definition of $\otimes$, that for all $n \in \mathbb{N}, \mu_{b} \in M_{n}^{b}, c \in[0,1]$ and $(A, B) \in Q_{n}, I_{b}\left(\mu_{b}, c \cdot \mathbf{1}_{(A, B)}\right)=\operatorname{sign}\left(\mu_{b}(A, B)\right)\left(c \otimes\left|\mu_{b}(A, B)\right|\right)$.

Due to Proposition 2 in Definition 7, axioms (U2), (U3) and (U4) can be equivalently substituted with the following axiom

(U6) there exists a semicopula $\otimes$ such that for all $n \in \mathbb{N}, \mu_{b} \in M_{n}^{b}, c \in[0,1]$ and $(A, B) \in Q_{n}, I_{b}\left(\mu_{b}, c \cdot \mathbf{1}_{(A, B)}\right)=\operatorname{sign}\left(\mu_{b}(A, B)\right)\left(c \otimes\left|\mu_{b}(A, B)\right|\right)$.

Note that the discrete bipolar Choquet, Shilkret and Sugeno integrals (1), (4) and (5) are $[-1,1]$-valued discrete bipolar universal integrals in the sense of Definition 7. Observe that the underlying semicopula $\otimes$ is the standard product in the case of the bipolar Choquet and Shilkret integrals, while $\otimes$ is the minimum (with neutral element $\beta=1$ ) for the bipolar Sugeno integral. Note also that for the bipolar Sugeno integral $S u_{b}\left(\mu_{b}, c \cdot \mathbf{1}_{(A, B)}\right)=c \otimes \mu_{b}(A, B)$ is the symmetric minimum.

The following theorem represents an alternative axiomatic characterization of the $[-1,1]$-valued discrete bipolar universal integral. 
Theorem 2 A function $I_{b}: \bigcup_{n \in \mathbb{N}}\left(M_{n}^{b} \times[-1,1]^{n}\right) \rightarrow[-1,1]$ is a $[-1,1]$-valued discrete bipolar universal integral if and only if it satisfies the following axioms:

(U6) there exists a semicopula $\otimes$ such that for all $n \in \mathbb{N}, \mu_{b} \in M_{n}^{b}, c \in[0,1]$ and $(A, B) \in Q_{n}, I_{b}\left(\mu_{b}, c \cdot \mathbf{1}_{(A, B)}\right)=\operatorname{sign}\left(\mu_{b}(A, B)\right)\left(c \otimes\left|\mu_{b}(A, B)\right|\right)$.

(U7) $I_{b}\left(\mu_{b_{1}}, \boldsymbol{x}_{1}\right) \geq I_{b}\left(\mu_{b_{2}}, \boldsymbol{x}_{2}\right)$ for all pairs $\left(\mu_{b_{1}}, \boldsymbol{x}_{1}\right) \in M_{n_{1}}^{b} \times[-1,1]^{n_{1}}$ and $\left(\mu_{b_{2}}, \boldsymbol{x}_{2}\right) \in$ $M_{n_{2}}^{b} \times[-1, \overline{1}]^{n_{2}}, n_{1}, n_{2} \in \mathbb{N}$, such that for all $\left.\left.t \in\right] 0,1\right]$,

$$
\mu_{b_{1}}\left(\left\{\boldsymbol{x}_{1} \geq t\right\},\left\{\boldsymbol{x}_{1} \leq-t\right\}\right) \geq \mu_{b_{2}}\left(\left\{\boldsymbol{x}_{2} \geq t\right\},\left\{\boldsymbol{x}_{2} \leq-t\right\}\right) .
$$

Proof. The sufficiency part is obvious, since it is easily checked that axiom (U7) implies axioms (U1) and (U5) while axiom (U6) implies (U2), (U3) and (U4). Now we prove the necessity part.

Let us suppose that axioms (U1)-(U5) hold. Axiom (U6) is true by Proposition 2 and, then, we only have to prove axiom (U7).

Let us suppose that for some $n_{1}, n_{2} \in \mathbb{N}, X_{1}=\left\{c_{1}, \ldots, c_{n_{1}}\right\}$ and $X_{2}=\left\{d_{1}, \ldots, d_{n_{2}}\right\}$ are two sets of criteria with $X_{n_{1}} \cap X_{n_{2}}=\emptyset$ and $X=X_{n_{1}} \cup X_{n_{2}}$. Let us indicate $\mathcal{Q}_{1}=\left\{(A, B) \mid A, B \in 2^{X_{1}}, A \cap B=\emptyset\right\}, \mathcal{Q}_{2}=\left\{(A, B) \mid A, B \in 2^{X_{2}}, A \cap B=\emptyset\right\}$ and $\mathcal{Q}=\left\{(A, B) \mid A, B \in 2^{X}, A \cap B=\emptyset\right\}$. If $\boldsymbol{x}=\left(x_{1}, \ldots, x_{n_{1}}\right)$ and $\boldsymbol{y}=\left(y_{1}, \ldots, y_{n_{2}}\right)$ let us consider $\boldsymbol{z}=\left(x_{1}, \ldots, x_{n_{1}}, y_{1}, \ldots, y_{n_{2}}\right) \in[-1,1]^{n_{1}+n_{2}}$ and the two bi-capacities $\bar{\mu}_{b}, \underline{\mu}_{b}: \mathcal{Q} \rightarrow[-1,1]$ defined for all $(A, B) \in \mathcal{Q}$ by

$$
\bar{\mu}_{b}(A, B)=\vee\left\{m_{1}\left(A \cap X_{1}, B \cap X_{1}\right), m_{2}\left(A \cap X_{2}, B \cap X_{2}\right)\right\}
$$

and

$$
\underline{\mu}_{b}(E)=\wedge\left\{m_{1}\left(A \cap X_{1}, B \cap X_{1}\right), m_{2}\left(A \cap X_{2}, B \cap X_{2}\right)\right\} .
$$

By axiom (U4) we have that $I_{b}\left(m_{1}, \boldsymbol{x}\right)=I_{b}\left(\bar{\mu}_{b}, \boldsymbol{z}\right)$ and $I_{b}\left(m_{2}, \boldsymbol{y}\right)=I_{b}\left(\mu_{b}, \boldsymbol{z}\right)$ and by axiom (U1), being $\bar{\mu}_{b} \geq \underline{\mu}_{b}, I_{b}\left(\bar{\mu}_{b}, \boldsymbol{z}\right) \geq I_{b}\left(\underline{\mu}_{b}, \boldsymbol{z}\right)$. Note that the condition $\bar{X}_{n_{1}}^{b} \cap X_{n_{2}}=\emptyset$ does not represent a limitation, since by axiom (U4), the value $I_{b}\left(\mu_{b}, \boldsymbol{x}\right)$ does not depend on the underlying space $\left(X_{n}, \mathcal{Q}_{n}\right)$ but only on the values of $\mu_{b}$ on the correspondent element of $\mathcal{Q}_{n}$.

\section{An illustrative example}

The following is an example of a bipolar universal integral, which is neither the Choquet nor Sugeno or Shilkret type. Let $I_{b}: \bigcup_{n \in \mathbb{N}}\left(M_{n}^{b} \times[-1,1]^{n}\right) \rightarrow[-1,1]$ be given by

$$
\left.\left.I\left(\mu_{b}, \boldsymbol{x}\right)=\sup ^{b i p}\left\{\frac{t \cdot \mu_{b}(\{\boldsymbol{x} \geq t\},\{\boldsymbol{x} \leq-t\})}{1-(1-t)\left(1-\left|\mu_{b}(\{\boldsymbol{x} \geq t\},\{\boldsymbol{x} \leq-t\})\right|\right)} \mid t \in\right] 0,1\right]\right\} .
$$

Note that (6) defines a bipolar universal integral. Indeed let us define for all $\left(\mu_{b}, \boldsymbol{x}\right) \in$ $M_{n}^{b} \times[-1,1]^{n}$ and for all $t \in[0,1], h^{\left(\mu_{b}, \boldsymbol{x}\right)}(t)=\mu_{b}(\{\boldsymbol{x} \geq t\},\{\boldsymbol{x} \leq-t\})$. Then if $\mu_{b} \geq \mu_{b}^{\prime}$ and $\boldsymbol{x} \geq \boldsymbol{x}^{\prime}$, we have $h^{\left(\mu_{b}, \boldsymbol{x}\right)} \geq h^{\left(\mu_{b}^{\prime}, \boldsymbol{x}^{\prime}\right)}$ and being the function $t \cdot h /[1-(1-t)(1-|h|)]$ non decreasing in $h \in \mathbb{R}$, we conclude that $I\left(\mu_{b}, \boldsymbol{x}\right) \geq I\left(\mu_{b}^{\prime}, \boldsymbol{x}^{\prime}\right)$ using the monotonicity of the bipolar supremum. Moreover for all $n \in \mathbb{N}, \mu_{b} \in M_{n}, c \in[0,1]$ and $(A, B) \in \mathcal{Q}_{n}$, 


$$
\begin{aligned}
I\left(\mu_{b}, c \cdot \mathbf{1}_{(A, B)}\right) & =\operatorname{sign}\left(\mu_{b}(A, B)\right) \frac{c \cdot\left|\mu_{b}(A, B)\right|}{1-(1-c)\left(1-\left|\mu_{b}(A, B)\right|\right)}= \\
& =\operatorname{sign}\left(\mu_{b}(A, B)\right)\left(c \otimes\left|\mu_{b}(A, B)\right|\right) .
\end{aligned}
$$

Here the semicopula $\otimes$ underlying the bipolar universal integral (6) is the Hamacher product

$$
a \otimes b=\left\{\begin{array}{cl}
0 & \text { if } a=b=0 \\
\frac{a \cdot b}{1-(1-a)(1-b)} & \text { else. }
\end{array}\right.
$$

Now let us compute this integral in the simple situation of $X_{2}=\{1,2\}$. In this case the functions we have to integrate can be identified with a two dimensional vector $(x, y) \in[-1,1]^{2}$ and we should define a bi-capacity on $\mathcal{Q}_{2}$. For example

$$
\begin{gathered}
\mu_{b}(\{1\}, \emptyset)=0.6, \quad \mu_{b}(\{2\}, \emptyset)=0.2, \quad \mu_{b}(\{1\},\{2\})=0.1, \\
\mu_{b}(\{2\},\{1\})=-0.1, \quad \mu_{b}(\emptyset,\{1\})=-0.3 \quad \text { and } \quad \mu_{b}(\emptyset,\{2\})=-0.5 .
\end{gathered}
$$

First let us consider the four cases $|x|=|y|$. If $x \geq 0$ :

$$
\begin{gathered}
I\left(\mu_{b},(x, x)\right)=x, \quad I\left(\mu_{b},(x,-x)\right)=\frac{0.1 x}{0.1+0.9 x} \\
I\left(\mu_{b},(-x, x)\right)=\frac{-0.1 x}{0.1+0.9 x} \text { and } I\left(\mu_{b},(-x,-x)\right)=-x .
\end{gathered}
$$

For all the other possible cases, we have the following formula

$$
I\left(\mu_{b},(x, y)\right)= \begin{cases}\bigvee^{b}\left\{y, \frac{0.6 x}{0.6+0.4 x}\right\} & x>y \geq 0 \\ \bigvee^{b}\left\{\frac{0.1|y|}{0.1+0.9|y|}, \frac{0.6 x}{0.6+0.4 x}\right\} & x \geq 0>y>-x \\ \bigvee^{b}\left\{\frac{0.1 x}{0.1+0.9 x}, \frac{-0.5|y|}{0.5+0.5|y|}\right\} & x \geq 0 \geq-x>y \\ \bigvee^{b}\left\{x, \frac{-0.5|y|}{0.5+0.5|y|}\right\} & 0>x>y \\ \bigvee^{b}\left\{x, \frac{0.2 y}{0.2+0.8 y}\right\} & y>x \geq 0 \\ \bigvee^{b}\left\{\frac{-0.1|x|}{0.1+0.9|x|}, \frac{0.2 y}{0.2+0.8 y}\right\} & y \geq 0>x>-y \\ \bigvee^{b}\left\{\frac{-0.1 y}{0.1+0.9 y}, \frac{-0.1|x|}{0.1+0.9|x|}\right\} & y \geq 0 \geq-y>x \\ \bigvee^{b}\left\{y, \frac{-0.3|x|}{0.3+0.7|x|}\right\} & 0>y>x .\end{cases}
$$

Observe that for arbitrary two bipolar integrals $I_{1}$ and $I_{2}$ related to semicopulas $\otimes_{1}$ and $\otimes_{2}$, respectively, also their convex combination is a bipolar universal integral, i.e., for each $\lambda \in[0,1], I=\lambda I_{1}+(1-\lambda) I_{2}$ is a bipolar universal integral related to semicopula $\otimes=\lambda \otimes_{1}+(1-\lambda) \otimes_{2}$. For example, convex combinations of Sugeno and Choquet bipolar integrals can be useful when fitting bipolar integrals to some real data, forming a parametric class of bipolar universal integrals. 


\section{Bipolar universal integrals and axiomatic foundation in terms of aggregation functions}

Suppose $I_{b}: \bigcup_{n \in \mathbb{N}}\left(M_{n}^{b} \times[-1,1]^{n}\right) \rightarrow[-1,1]$ is a $[-1,1]$-valued discrete bipolar universal integral and for some $n \in \mathbb{N}$, consider a fixed $\mu_{b}^{*} \in M_{n}^{b}$. The function $I_{\mu_{b}^{*}}:[-1,1]^{n} \rightarrow[-1,1]$ defined by $I_{\mu_{b}^{*}}(\boldsymbol{x})=I_{b}\left(\mu_{b}^{*}, \boldsymbol{x}\right)$ for all $\boldsymbol{x} \in[-1,1]^{n}$, is a bipolar aggregation function from $\mathcal{A}_{[-1,1]^{n}}$. Thus any $[-1,1]$-valued discrete bipolar universal integral can be viewed as a family of aggregations functions, $I_{\mu_{b}}(\cdot) \in \bigcup_{n \in \mathbb{N}} \mathcal{A}_{[-1,1]^{n}}$, one for each bi-capacity $\mu_{b} \in \bigcup_{n \in \mathbb{N}} M_{n}^{b}$. In this section we shall characterize [-1,1]-valued discrete bipolar universal integrals, starting from a family of aggregation functions of $\bigcup_{n \in \mathbb{N}} \mathcal{A}_{[-1,1]^{n}}$ satisfying some desired properties.

Consider a family $\mathcal{F}_{b} \subseteq \bigcup_{n \in \mathbb{N}} \mathcal{A}_{[-1,1]^{n}}$ with $\mathcal{F}_{b} \neq \emptyset$ and consider the following axioms on $\mathcal{F}_{b}$ :

(B1) For all $f \in \mathcal{F}_{b} \cap \mathcal{A}_{[-1,1]^{n_{1}}}, g \in \mathcal{F}_{b} \cap \mathcal{A}_{[-1,1]^{n_{2}}}$ and $\boldsymbol{x} \in[-1,1]^{n_{1}}, \boldsymbol{y} \in[-1,1]^{n_{2}}$, $n_{1}, n_{2} \in \mathbb{N}$, such that for all $\left.\left.t \in\right] 0,1\right]$

$$
f\left(\mathbf{1}_{(\{\boldsymbol{x} \geq t\},\{\boldsymbol{x} \leq-t\})}\right) \geq g\left(\mathbf{1}_{\{(\boldsymbol{y} \geq t\},\{\boldsymbol{y} \leq-t\})}\right),
$$

then $f(\boldsymbol{x}) \geq g(\boldsymbol{y})$.

(B2) Every $f \in \mathcal{F}_{b}$ is idempotent, i.e., for all $n \in \mathbb{N}, c \in[-1,1]$ and $f \in \mathcal{F}_{b} \cap \mathcal{A}_{[-1,1]^{n}}$, $f\left(c \cdot \mathbf{1}_{\left(X_{n}, \emptyset\right)}\right)=-f\left(c \cdot \mathbf{1}_{\left(\emptyset, X_{n}\right)}\right)=c$.

(B3) For all $n \in \mathbb{N}$ and for all $\mu_{b} \in M_{n}^{b}$ there exists $f \in \mathcal{F}_{b} \cap \mathcal{A}_{[-1,1]^{n}}$ such that $f\left(\mathbf{1}_{(A, B)}\right)=\mu_{b}(A, B)$ for all $(A, B) \in \mathcal{Q}_{n}$.

(B4) For all $n_{1}, n_{2} \in \mathbb{N}, f \in \mathcal{F}_{b} \cap \mathcal{A}_{[-1,1]^{n_{1}}}, g \in \mathcal{F}_{b} \cap \mathcal{A}_{[-1,1]^{n_{2}}}$, and $\left(A_{1}, B_{1}\right) \in$ $\mathcal{Q}_{n_{1}},\left(A_{2}, B_{2}\right) \in \mathcal{Q}_{n_{2}}$, such that

$$
f\left(\mathbf{1}_{\left(A_{1}, B_{1}\right)}\right)=-g\left(\mathbf{1}_{\left(A_{2}, B_{2}\right)}\right)
$$

then for all $c \in[0,1]$,

$$
f\left(c \cdot \mathbf{1}_{\left(A_{1}, B_{1}\right)}\right)=-g\left(c \cdot \mathbf{1}_{\left(A_{2}, B_{2}\right)}\right) .
$$

Observe that the above introduced axioms (B1)-(B4) are independent.

Theorem 3 Axioms (B1)-(B4) hold for a family $\mathcal{F}_{b} \subseteq \bigcup_{n \in \mathbb{N}} \mathcal{A}_{[-1,1]^{n}}$, if and only if there exists a $[-1,1]$-valued discrete bipolar universal integral $I_{b}$ with a semicopula $\otimes$ such that, for all $n \in \mathbb{N}$ and $f \in \mathcal{F}_{b} \cap \mathcal{A}_{[-1,1]^{n}}$ there exists a bi-capacity $\mu_{b}(f) \in M_{n}^{b}$ such that

$$
f(\boldsymbol{x})=I_{b}\left(\mu_{b}(f), \boldsymbol{x}\right) \text { for all } \boldsymbol{x} \in[-1,1]^{n} .
$$

More precisely,

(a) for all $n \in \mathbb{N}, f \in \mathcal{F}_{b} \cap \mathcal{A}_{[-1,1]^{n}}$ and $(A, B) \in \mathcal{Q}_{n}, \mu_{b}(f)(A, B)=f\left(\boldsymbol{1}_{(A, B)}\right)$;

(b) for all $\left.x, y \in[0,1], x \otimes y=f\left(x \mathbf{1}_{(A, B}\right)\right)$, with $f\left(\mathbf{1}_{(A, B)}\right)=y,(A, B) \in \mathcal{Q}_{n}$ and $f \in \mathcal{F}_{b} \cap \mathcal{A}_{[-1,1]^{n}}$ for some $n \in \mathbb{N}$. 
Proof. First we prove the sufficiency part, i.e. we suppose that axioms (B1)-(B4) hold for the family $\mathcal{F}_{b}$ and we prove the existence of a $[-1,1]$-discrete bipolar universal integral satisfying equation (9).

Our first step is to prove that for any $n \in \mathbb{N}$ and $f \in \mathcal{F}_{b} \cap \mathcal{A}_{[-1,1]^{n}}$ we can define a bi-capacity by means of the relationship

$$
\mu_{b}(f)(A, B)=f\left(\mathbf{1}_{(A, B)}\right) \text { for all }(A, B) \in \mathcal{Q}_{n} .
$$

Being $f$ a bipolar aggregation function, $\mu_{b}(f)\left(X_{n}, \emptyset\right)=f\left(\mathbf{1}_{\left(X_{n}, \emptyset\right)}\right)=1, \mu_{b}(f)\left(\emptyset, X_{n}\right)=$ $f\left(\mathbf{1}_{\left(\emptyset, X_{n}\right)}\right)=-1$ and $\mu_{b}(f)(\emptyset, \emptyset)=f\left(\mathbf{1}_{(\emptyset, \emptyset)}\right)=0$. Moreover, if $(A, B),(C, D) \in \mathcal{Q}_{n}$ with $(A, B) \precsim(C, D)$ then $\mathbf{1}_{(A, B)} \preceq \mathbf{1}_{(C, D)}$ and by monotonicity of $f$ we have that $\mu_{b}(f)(A, B)=f\left(\mathbf{1}_{(A, B)}\right) \leq f\left(\mathbf{1}_{(C, D)}\right)=\mu_{b}(f)$. Thus for any $f \in \mathcal{F}_{b}, \mu_{b}(f)$ is a bicapacity.

Our second step is to prove that a semicopula is defined by setting for all $x, y \in$ $\left.[0,1], x \otimes y=f\left(x \mathbf{1}_{(A, B}\right)\right)$, with $f\left(\mathbf{1}_{(A, B)}\right)=y,(A, B) \in \mathcal{Q}_{n}$ and $f \in \mathcal{F}_{b} \cap \mathcal{A}_{[-1,1]^{n}}$ for some $n \in \mathbb{N}$. First of all observe that the definition of $\otimes$ is well posed. Indeed, for any $b \in[0,1]$ there exist $n \in \mathbb{N}, f \in \mathcal{F}_{b} \cap \mathcal{A}_{[-1,1]^{n}}$ and $(A, B),(C, D) \in \mathcal{Q}_{n}$ such that $f\left(\mathbf{1}_{(A, B)}\right)=b$ and $f\left(\mathbf{1}_{(C, D)}\right)=-b$ by (B3) (see also the beginning of the proof of Proposition 2). Moreover, if for some $f \in \mathcal{F}_{b} \cap \mathcal{A}_{[-1,1]^{n_{1}}}, g \in \mathcal{F}_{b} \cap \mathcal{A}_{[-1,1]^{n_{2}}}$ and $c \in[0,1]$ we have that $f\left(\mathbf{1}_{(A, B)}\right)=g\left(\mathbf{1}_{(C, D)}\right)$ with $(A, B) \in \mathcal{Q}_{n_{1}}$ and $(C, D) \in \mathcal{Q}_{n_{2}}$, then $f\left(c \mathbf{1}_{(A, B)}\right)=g\left(c \mathbf{1}_{(C, D)}\right)$ by $(\mathrm{B} 1)$, because

$$
\left.\left.f\left(\mathbf{1}_{\left(\left\{c \mathbf{1}_{(A, B)} \geq t\right\},\left\{c \mathbf{1}_{(A, B)} \leq-t\right\}\right)}\right)=g\left(\mathbf{1}_{\left(\left\{c \mathbf{1}_{(C, D)} \geq t\right\},\left\{c \mathbf{1}_{(C, D)} \leq-t\right\}\right)}\right) \text { for all } t \in\right] 0,1\right] .
$$

Thus, $\otimes$ is well defined and we must prove that it is a semicopula.

To prove that $\otimes$ is nondecreasing, let us consider $x_{1}, x_{2}, y_{1}, y_{2} \in[0,1]$ such that $x_{1} \leq x_{2}$ and $y_{1} \leq y_{2}$. By definition there exist $f \in \mathcal{F}_{b} \cap \mathcal{A}_{[-1,1]^{n_{1}}}, g \in \mathcal{F}_{b} \cap \mathcal{A}_{[-1,1]^{n_{2}}}$ such that we have that $x_{1} \otimes y_{1}=f\left(x_{1} \mathbf{1}_{(A, B)}\right)$ with $(A, B) \in \mathcal{Q}_{n_{1}}$ such that $f\left(\mathbf{1}_{(A, B)}\right)=y_{1}$ and $x_{2} \otimes y_{2}=g\left(x_{2} \mathbf{1}_{(C, D)}\right)$ with $(C, D) \in \mathcal{Q}_{n_{2}}$ such that $g\left(\mathbf{1}_{(C, D)}\right)=y_{2}$. It results $f\left(x_{1} \mathbf{1}_{(A, B)}\right) \leq g\left(x_{2} \mathbf{1}_{(C, D)}\right)$ by $(\mathrm{B} 1)$, because

$$
\left.\left.f\left(\mathbf{1}_{\left(\left\{x_{1} \mathbf{1}_{(A, B)} \geq t\right\},\left\{x_{1} \mathbf{1}_{(A, B)} \leq-t\right\}\right)}\right) \leq g\left(\mathbf{1}_{\left(\left\{x_{2} \mathbf{1}_{(C, D)} \geq t\right\},\left\{x_{2} \mathbf{1}_{(C, D)} \leq-t\right\}\right)}\right) \text { for all } t \in\right] 0,1\right] .
$$

Now we prove that 1 is a neutral element for $\otimes$. For all $a \in[0,1]$ we have that for any $f \in \mathcal{F}_{b} \cap \mathcal{A}_{[-1,1]^{n}}, a \otimes 1=f\left(a \mathbf{1}_{\left(X_{n}, \emptyset\right)}\right)=a$ by idempotency of $f$. Moreover, by definition of $\otimes$ we have that $1 \otimes a=f\left(1 \cdot \mathbf{1}_{(A, B)}\right)=a$ for some $f \in \mathcal{F}_{b} \cap \mathcal{A}_{[-1,1]^{n}}$ and $(A, B) \in \mathcal{Q}_{n}$. We conclude that $\otimes$ is a semicopula.

Let us note that, due to axiom (B4), we could equivalently define $\otimes$ by setting for all $\left.x, y \in[0,1], \otimes(x, y)=-f\left(x \mathbf{1}_{(A, B}\right)\right)$, with $f\left(\mathbf{1}_{(A, B)}\right)=-y$ with $(A, B) \in \mathcal{Q}_{n}$, $f \in \mathcal{F}_{b} \cap \mathcal{A}_{[-1,1]^{n}}$ for some $n \in \mathbb{N}$.

For any $n \in \mathbb{N}$ and $f \in \mathcal{F}_{b} \cap \mathcal{A}_{[-1,1]^{n}}$ we have defined a bi-capacity by means of $\mu_{b}(f)(A, B)=f\left(\mathbf{1}_{(A, B)}\right)$ for all $(A, B) \in \mathcal{Q}_{n}$. On the converse for any $n \in \mathbb{N}$ and $\mu_{b} \in M_{n}^{b}$, by (B3) there exists an $f \in \mathcal{F}_{b} \cap \mathcal{A}_{[-1,1]^{n}}$ such that $f\left(\mathbf{1}_{(A, B)}\right)=\mu_{b}(A, B)$ for all $(A, B) \in \mathcal{Q}_{n}$. Axiom (B1) ensures that function $f$ is unique, indeed: suppose that $f\left(\mathbf{1}_{(A, B)}\right)=g\left(\mathbf{1}_{(A, B)}\right)$ for all $(A, B) \in \mathcal{Q}_{n}$, then by (B1) for all $\boldsymbol{x} \in[-1,1]^{n}$ we have that $f\left(\mathbf{1}_{(\{\boldsymbol{x} \geq t\},\{\boldsymbol{x} \leq-t\})}\right)=g\left(\mathbf{1}_{(\{\boldsymbol{x} \geq t\},\{\boldsymbol{x} \leq-t\})}\right)$ for all $\left.\left.t \in\right] 0,1\right]$ and then $f(\boldsymbol{x})=g(\boldsymbol{x})$. Due to the proved uniqueness we indicate with $f_{\mu_{b}}$ the function from $\mathcal{F}_{b}$ such that $f_{\mu_{b}}\left(\mathbf{1}_{(A, B)}\right)=\mu_{b}(A, B)$ for all $(A, B) \in \mathcal{Q}_{n}$ and, obviously, $\mu_{b}\left(f_{\mu_{b}^{*}}\right)=\mu_{b}^{*}$.

Aggregation functions from $\mathcal{F}_{b}$ define a function $I_{b}: \bigcup_{n \in \mathbb{N}}\left(M_{n}^{b} \times[-1,1]^{n}\right) \rightarrow[-1,1]$ by means of $I_{b}\left(\mu_{b}, \boldsymbol{x}\right)=f_{\mu_{b}}(\boldsymbol{x})$ for all $\left(\mu_{b}, \boldsymbol{x}\right) \in \bigcup_{n \in \mathbb{N}}\left(M_{n}^{b} \times[-1,1]^{n}\right)$. Let us prove that the function $I_{b}$ is a bipolar universal integral with respect to semicopula $\otimes$. 
We use the characterization of Theorem 2, then we have to prove that $I_{b}$ satisfies axioms (U6) and (U7).

For all $n \in \mathbb{N}, \mu_{b} \in M_{n}^{b}, c \in[0,1]$ and $(A, B) \in Q_{n}, I_{b}\left(\mu_{b}, c \cdot \mathbf{1}_{(A, B)}\right)=f_{\mu_{b}}\left(c \cdot \mathbf{1}_{(A, B)}\right)$. If $f_{\mu_{b}}\left(\mathbf{1}_{(A, B)}\right)=\mu_{b}(A, B) \geq 0$, then by definition of $\otimes, f_{\mu_{b}}\left(c \cdot \mathbf{1}_{(A, B)}\right)=c \otimes \mu_{b}(A, B)$, while if $f_{\mu_{b}}\left(\mathbf{1}_{(A, B)}\right)=\mu_{b}(A, B)<0$, then, again by definition of $\otimes$ and by axiom (B4), $f_{\mu_{b}}\left(c \cdot \mathbf{1}_{(A, B)}\right)=-c \otimes\left|\mu_{b}(A, B)\right|$. Thus, in any case $I_{b}\left(\mu_{b}, c \cdot \mathbf{1}_{(A, B)}\right)=\operatorname{sign}\left(\mu_{b}(A, B)\right)(c \otimes$ $\left.\left|\mu_{b}(A, B)\right|\right)$ and $(\mathrm{U} 6)$ is proved.

Consider $\left(\mu_{b_{1}}, \boldsymbol{x}_{1}\right) \in M_{n_{1}}^{b} \times[-1,1]^{n_{1}}$ and $\left(\mu_{b_{2}}, \boldsymbol{x}_{2}\right) \in M_{n_{2}}^{b} \times[-1,1]^{n_{2}}, n_{1}, n_{2} \in \mathbb{N}$, such that for all $t \in] 0,1], \mu_{b_{1}}\left(\left\{\boldsymbol{x}_{1} \geq t\right\},\left\{\boldsymbol{x}_{1} \leq-t\right\}\right) \geq \mu_{b_{2}}\left(\left\{\boldsymbol{x}_{2} \geq t\right\},\left\{\boldsymbol{x}_{2} \leq-t\right\}\right)$. By definition this means $f_{\mu_{b_{1}}}\left(\boldsymbol{x}_{1}\right)=I_{b}\left(\mu_{b_{1}}, \boldsymbol{x}_{1}\right) \geq f_{\mu_{b_{2}}}\left(\boldsymbol{x}_{2}\right)=I_{b}\left(\mu_{b_{2}}, \boldsymbol{x}_{2}\right)$, which proves (U7) and concludes the sufficiency part.

Now we prove the necessity part. Suppose that $I_{b}: \bigcup_{n \in \mathbb{N}}\left(M_{n}^{b} \times[-1,1]^{n}\right) \rightarrow[-1,1]$ is a bipolar universal integral with respect to some semicopula $\otimes$. For fixed $n \in \mathbb{N}$, $\mu_{b} \in M_{n}^{b}$ the $I_{\mu_{b}}:[-1,1]^{n} \rightarrow[-1,1]$ defined by $I_{\mu_{b}}(\boldsymbol{x})=I_{b}\left(\mu_{b}, \boldsymbol{x}\right)$ for all $\boldsymbol{x} \in[-1,1]^{n}$, is a bipolar aggregation function. Let us prove that the family of aggregation functions $\mathcal{F}_{b}=\left\{I_{\mu_{b}} \mid \mu_{b} \in \bigcup_{n \in \mathbb{N}} M_{n}^{b}\right\}$ satisfies axioms (B1)-(B4). Since $I_{b}$ is a discrete universal integral, it satisfies axioms (U6) and (U7) of Theorem 2 and then (B1) follows directly by definition of $\mathcal{F}_{b}$ and by (U7) while (B2), (B3) and (B4) follow by definition of $\mathcal{F}_{b}$ and by (U6).

\section{Conclusions}

In this paper we have defined and axiomatically characterized the $[-1,1]$-valued discrete bipolar universal integral, thus providing a common frame including the discrete bipolar Choquet, Shilkret and Sugeno integrals. Moreover, an axiomatic characterization of bipolar universal integrals in the framework of bipolar aggregation functions was introduced, too. We believe that the concept of bipolar universal integral will allow new theoretical developments, where the bipolarity of choices is involved, both in MCDA as well as in decision under risk and uncertainty.

\section{Acknowledgment}

This work was supported by grants GACR P 402/11/0378 and VEGA 1/0171/12. 


\section{Bibliography}

[1] M. Allais. Le comportement de l'homme rationnel devant le risque: Critique des postulats et axiomes de l'école Américaine. Econometrica, 21(4):503-546, 1953.

[2] B. Bassan and F. Spizzichino. Relations among univariate aging, bivariate aging and dependence for exchangeable lifetimes. Journal of Multivariate Analysis, 93(2):313-339, 2005.

[3] S. Bodjanova and M. Kalina. Sugeno and Shilkret integrals, and T- and Sevaluators. In SISY 2009 - 7th International Symposium on Intelligent Systems and Informatics, pages 109-114, 2009.

[4] G. Choquet. Theory of Capacities. Annales de l'Institute Fourier (Grenoble), 5:131-295, 1953/54.

[5] D. Ellsberg. Risk, ambiguity, and the Savage axioms. The Quarterly Journal of Economics, 75(4):643-669, 1961.

[6] I. Gilboa. Expected utility with purely subjective non-additive probabilities. Journal of Mathematical Economics, 16(1):65-88, 1987.

[7] M. Grabisch. The application of fuzzy integrals in multicriteria decision making. European Journal of Operational Research, 89(3):445-456, 1996.

[8] M. Grabisch. The symmetric Sugeno integral. Fuzzy Sets and Systems, 139(3):473490, 2003.

[9] M. Grabisch. The Möbius transform on symmetric ordered structures and its application to capacities on finite sets. Discrete Mathematics, 287(1-3):17-34, 2004.

[10] M. Grabisch, S. Greco, and M. Pirlot. Bipolar and bivariate models in multicriteria decision analysis: Descriptive and constructive approaches. Int. J. Intell. Syst., 23(9):930-969, 2008.

[11] M. Grabisch and C. Labreuche. Bi-capacities-I: definition, Möbius transform and interaction. Fuzzy Sets and Systems, 151(2):211-236, 2005.

[12] M. Grabisch and C. Labreuche. Bi-capacities-II: the Choquet integral. Fuzzy Sets and Systems, 151(2):237-259, 2005.

[13] M. Grabisch and C. Labreuche. Fuzzy measures and integrals in MCDA. Multiple criteria decision analysis: state of the art surveys, pages 563-604, 2005.

[14] M. Grabisch and C. Labreuche. A decade of application of the Choquet and Sugeno integrals in multi-criteria decision aid. 4OR: A Quarterly Journal of Operations Research, 6(1):1-44, 2008.

[15] M. Grabisch, J.L. Marichal, R. Mesiar, and E. Pap. Aggregation Functions (Encyclopedia of Mathematics and its Applications). Cambridge University Press, Cambridge, 2009.

[16] S. Greco. Generalizing again the Choquet integral: the profile dependent Choquet integral. In D. Dubois, M. Grabisch, R. Mesiar, and E. P. Klement, editors, Decision Theory: Qualitative and Quantitative Approaches, pages 66-79. Linz Seminar on Fuzzy Set Theory, 2011.

[17] S. Greco, B. Matarazzo, and S. Giove. The Choquet integral with respect to a level dependent capacity. Fuzzy Sets and Systems, 175(1):1-35, 2011.

[18] S. Greco, B. Matarazzo, and R. Slowinski. Bipolar Sugeno and Choquet integrals. In B. De Baets, J. Fodor, and G. Pasi, editors, EURO Working Group on Fuzzy Sets, pages 191-196. Workshop on Informations Systems (EUROFUSE 2002), Varenna, Italy, 2002. 
[19] S. Greco, R. Mesiar, and F. Rindone. The bipolar universal integral. Advances in Computational Intelligence, pages 360-369, 2012.

[20] S. Greco and F. Rindone. Bipolar fuzzy integrals. Fuzzy Sets and Systems, 220:2133, 2013.

[21] S. Greco and F. Rindone. The bipolar Choquet integral representation. Theory and Decision (under revision), 2013.

[22] D. Kahneman and A. Tversky. Prospect theory: An analysis of decision under risk. Econometrica, 47(2):263-291, 1979.

[23] E.P. Klement, A. Kolesárová, R. Mesiar, and A. Stupňanová. Universal Integrals Based on Level Dependent Capacities. In IFSA-EUSFLAT. Citeseer, 2009.

[24] E.P. Klement, A. Kolesárová, R. Mesiar, and A. Stupňanová. A generalization of universal integrals by means of level dependent capacities. Knowledge-Based Systems, 38:14-18, 2013.

[25] E.P. Klement and R. Mesiar. Discrete Integrals and Axiomatically Defined Functionals. Axioms, 1(1):9-20, 2012.

[26] E.P. Klement, R. Mesiar, and E. Pap. A universal integral as common frame for Choquet and Sugeno integral. IEEE Transactions on Fuzzy Systems, 18(1):178$187,2010$.

[27] R.D. Luce. Binary Gambles of a Gain and a Loss: an Understudied Domain. Mathematical utility theory: utility functions, models, and applicaitons in the social sciences, 8:181-202, 1999.

[28] R.D. Luce. Utility of Gains and Losses: Measurement-Theoretical, and Experimental Approaches. Lawrence Erlbaum Associates, 2000.

[29] R. Mesiar, A. Mesiarová-Zemánková, and K. Ahmad. Level-dependent Sugeno integral. IEEE Transactions on Fuzzy Systems, 17(1):167-172, 2009.

[30] R. Mesiar, A. Mesiarová-Zemánková, and K. Ahmad. Discrete Choquet integral and some of its symmetric extensions. Fuzzy Sets and Systems, 184(1):148-155, 2011.

[31] D. Schmeidler. Integral representation without additivity. Proceedings of the American Mathematical Society, 97(2):255-261, 1986.

[32] D. Schmeidler. Subjective probability and expected utility without additivity. Econometrica, 57(3):571-587, 1989.

[33] N. Shilkret. Maxitive measure and integration. In Indagationes Mathematicae (Proceedings), volume 74, pages 109-116. Elsevier, 1971.

[34] C. Starmer. Developments in non-expected utility theory: The hunt for a descriptive theory of choice under risk. Journal of economic literature, 38(2):332-382, 2000 .

[35] M. Sugeno. Theory of fuzzy integrals and its applications. Ph.D. Thesis, Tokyo Institute of Technology, 1974.

[36] A. Tversky and D. Kahneman. The framing of decisions and the psychology of choice. Science, 211(4481):453, 1981.

[37] A. Tversky and D. Kahneman. Advances in prospect theory: Cumulative representation of uncertainty. Journal of Risk and uncertainty, 5(4):297-323, 1992.

[38] J. Von Neumann, O. Morgenstern, H.W. Kuhn, and A. Rubinstein. Theory of games and economic behavior. Princeton university press, Princeton, NJ, 1947.

[39] G. Wu and A.B. Markle. An empirical test of gain-loss separability in prospect theory. Management Science, 54(7):1322-1335, 2008. 